\title{
MODELOS DE PREDICCIÓN DEL IMPACTO Y EVOLUCIÓN DEL COVID-19 EN REPÚBLICA DOMINICANA
}

\section{Prediction models of the impact and evolution of COVID-19 in the Dominican Republic}

\section{Edian F. Franco}

Área de Ciencias Básicas y Ambientales, Instituto Tecnológico de Santo Domingo (INTEC). Santo Domingo. Departamento de Biotecnología, Instituto de Ciencias Biológicas, Universidad Federal de Para, Belém, Para, Brasil. ORCID: 0000-0001-9715-9437 Correo-e: edianfranco@ufpa.br, edian.franco@intec.edu.do

\author{
Víctor V. Calderón \\ Departamento de Ciencias Básicas y Ambientales, \\ Instituto Tecnológico de Santo Domingo (NTEC), \\ Santo Domingo, República Dominicana \\ ORCID: 0000-0002-8537-1358 \\ Correo-e: victorvirgilioc@gmail.com \\ Rommel T. Ramos \\ Departamento de Biotecnología, Instituto de Ciencias \\ Biológicas, Universidad Federal de Para, Belém, Para, \\ Brasil. ORCID: 0000-0002-8032-1474 \\ Correo-e: rommelthiago@gmail.com
}

Recibido: 5/5/2020 • Aprobado: 17/6/2020

Cómo citar: F. Franco, E., Calderón, V. V., \& Ramos, R. T. (2020). Modelos de predicción del impacto y evolución del COVID-19 en República Dominicana. Ciencia, Ambiente y Clima, 3(1), 5-21. Doi: https://doi.org/10.22206/cac.2020.v3i1.pp5-21

\section{Resumen}

Después de su descubrimiento en China, en diciembre de 2019, el virus SARS-CoV-2 causante de la enfermedad COVID-19, comenzó a propagarse por la mayoría de los países del mundo, provocando una cantidad importante de infectados y muertos, situación que ha generado una gran presión en los sistemas de salud y gobiernos de los diferentes países, que en muchos casos no contaban con los recursos e infraestructuras para enfrentar una pandemia provocada por el virus, como fue el caso de República Dominicana. Usando los datos públicos del avance de la pandemia en la Republica Dominicana, desde el 1 de marzo hasta el 30 de abril, se modeló y pronosticó la posible evolución del COVID-19 en este país, utilizando de tres modelos matemáticos SIR, SEIR-Extendido y log-lineal. Estos modelos permitieron proyectar los diferentes escenarios de contagios y evolución de la enfermedad, además de pronosticar el pico y el descenso de la curva de casos. Según los resultados obtenidos, el país se encontraba en un proceso de crecimiento exponencial de los contagios;

\begin{abstract}
After its discovery in China, in December 2019, the SARS-CoV-2 virus causing the COVID-19 disease began to spread through countries around the world, causing a significant number of infected and dead. This situation has put high pressure on the health systems and governments of the different countries, which in many cases did not have the resources and infrastructure to face a pandemic caused by the virus, as was the case in the Dominican Republic. Using the public data of the advance of the pandemic in the Dominican Republic, from March 1 to April 30, the possible evolution of COVID-19 in this country was modeled and predicted, using three mathematical models SIR, SEIR-Extended, and log-linear. These models made it possible to project the different scenarios of infection and disease evolution, in addition to calculating the peak and decrease in the case curve. According to the results obtained, the country presented an exponential growth process of infections, with the peak of infections being projected to occur between the second
\end{abstract}


el pico de los contagios fue pronosticado ocurriría entre la segunda mitad del mes de mayo y la primera mitad del mes de junio, con un estimado de infectados entre 100,000 y 200,000 individuos. Los resultados obtenidos en este estudio permiten obtener una idea de cuál podría ser el impacto humano y social del COVID-19 en la República Dominicana.

Palabras clave: SARS-CoV-2; COVID-19; República Dominicana; modelos matemáticos; pronóstico; SIR; SEIR.

\section{Introducción}

En el último trimestre del año 2019, en la ciudad de Wuhan, provincia de Hubei, China, fueron reportados un gran número de casos de afecciones respiratorias de las vías inferiores, que hasta ese momento eran de origen idiopático. En muchos de los pacientes, estas afecciones estaban causando un cuadro clínico caracterizado por una dificultad respiratoria aguda, similar al síndrome respiratorio agudo severo (SARS). A partir de estudios de secuenciación de nueva generación de muestras obtenidas de pacientes chinos, se demostró que se trataba de un nuevo coronavirus, designado como SARS-CoV-2, que es el factor causal de la enfermedad COVID-19 (Wang, Horby, Hayden, \& Gao, 2020; Zhu et al., 2020).

El virus pudo tener un origen zoonótico (Andersen, Rambaut, Lipkin, Holmes, \& Garry, 2020), debido a que muchos de los primeros casos de pacientes reportados en China estaban relacionados al mercado mojado de Huana, donde existe la comercialización de diferentes tipos de animales silvestres (Hui et al., 2020; Zhou et al., 2020). El SARS-CoV-2 presenta una alta similitud con otros coronavirus como el SARS-CoV, identificado en China en 2002, causante del síndrome respiratorio agudo grave (SARS). Algunas hipótesis sugieren que el murciélago (Rhinolophus affinis) es el reservorio progenitor, debido a que el virus RaTG13 muestreado en este quiróptero, presentó una similitud de $\sim 96 \%$ con el virus del half of May and the first half of June, with an estimated infected between 100,000 and 200,000 individuals. The results obtained in this study allow us to get an idea of what COVID-19 could have in human and social impact in the Dominican Republic.

Keywords: SARS-CoV-2; COVID-19; Dominican Republic; Mathematical Models; estimation; SIR; SEIR.

SARS-CoV-2 (Zhou et al., 2020). Otra hipótesis sugiere que el virus pudiera tener a los pangolines de Malasia (Manis javanica) como organismos reservorios del virus, debido a que se han identificados coronavirus similares al SARS-CoV-2, en animales de esta especie vendidos en los mercados de China (Forster, Forster, Renfrew, \& Forster, 2020; Wu et al., 2020).

El COVID-19 es la enfermedad infecciosa causada por el SARS-CoV-2, esta se caracteriza por síntomas clínicos como fiebre, tos seca y fatiga. También se han descritos casos de pacientes que relatan dolor muscular, dolor de cabeza o confusión, dolor abdominal, náuseas, vómitos y diarrea. Entre los casos más severos de pacientes que han sido hospitalizados, se han observado linfógena, hipercitoquinemia, coagulación intravasal diseminada y dańo renal agudo (Chan et al., 2020; Chen et al., 2020; Huang et al., 2020; Wang et al., 2020). Los pacientes que desarrollan el síndrome respiratorio agudo tienen un cuadro clínico grave, que tiende a empeorar en un periodo corto de tiempo, provocando la muerte por insuficiencia orgánica múltiple (Tang, Wang et al., 2020; Zhao, Lin et al., 2020).

En la actualidad el virus se ha extendido a más de 100 países en todo el mundo, con una tasa de contagio $\left(\mathrm{R}_{0}\right)$ estimada entre 1.5 y 6.8 (Liu, Gayle, Wilder-Smith, \& Rocklöv, 2020; Viceconte \& Petrosillo, 2020; WHO, 2020b), provocando $\sim 3,000,000$ de personas infectadas y $\sim 233,792$ a 
nivel mundial al 30 de abril de 2020 (Dong, Du, \& Gardner, 2020). Reportes han informado que la tasa de letalidad de los pacientes hospitalizado está entre $2.3 \%$ y $11.5 \%$ (Chen et al., 2020), tasa que varía de país a país y depende de la infraestructura sanitaria disponible para la atención de los pacientes con esta enfermedad (Zhao, Musa et al., 2020).

En la República Dominicana, el primer caso de COVID-19 fue confirmado el primero de marzo de 2020, un paciente masculino de 62 ańos de edad, de nacionalidad italiana, quien habría ingresado al país nueve días antes (Organización Panamericana de la Salud, 2020). Desde esa fecha comenzó el proceso de expansión del agente infeccioso dentro de país, situación que obligó a las autoridades a tomar medidas con la finalidad de contener la propagación de virus. En la actualidad, el país está entre la lista de los más afectado en América Latina y ocupa el puesto número seis en la región (Rios Montanez, 2020) y el número 43 a nivel mundial (datos al 29/04/2020) (Dong et al., 2020), en relación con la cantidad de infectados que han sido confirmados. El país reporta a finales de abril de 2020 que había sobrepasado los 6,500 infectados y los 300 muertos por causa de la enfermedad, número que puede ser mayor debido a los casos asintomáticos que no son reportados y la cantidad limitada de pruebas que han sido realizadas.

Diversos esfuerzos están siendo realizados por la comunidad científica con la intención de modelar y predecir la evolución y el impacto de la pandemia en los diferentes países y regiones (Amira et al., 2020; Sanche et al., 2020; Tang, Bragazzi et al., 2020; Tang, Wang et al., 2020; Zhao, Musa et al., 2020). Una de las herramientas que puede ayudar en esta tarea son los modelos matemáticos y computacionales, que permiten generar proyecciones de los diferentes escenarios de la pandemia, lo que puede ayudar en la planificación de las acciones, recursos e infraestructuras; además, permiten probar diferentes hipótesis, y el descarte de soluciones poco realistas, o muy optimistas.
La Organización Mundial de la Salud (OMS), ha reconocido que los modelos matemáticos, estadísticos y computacionales pueden desempeñar un papel clave para proporcionar información, basada en evidencias, a los responsables de tomar decisiones en materia de políticas de salud públicas. Estos modelos pueden ayudar a comprender qué tan transmisible es la enfermedad; cuándo serán más altas las tasas de infecciones; qué tan grave es la infección y qué tan efectivas han sido las intervenciones (Tang, Bragazzi, et al., 2020).

En la actualidad, diversos modelos son usados para comprender las enfermedades infecciosas, como el modelo Susceptible-Infectious-Recovered (SIR) (Kermack \& McKendrick, 1927; Lin, Muthuraman, \& Lawley, 2010), utilizado para modelar enfermedades infecciosas que se propagan a través de gotitas y aerosoles como la gripe pandémica (Bootsma \& Ferguson, 2007), la gripe estacional (Markel et al., 2006), el SARS (Riley et al., 2003), y la viruela (Riley $\&$ Ferguson, 2006) y pueden ayudar a entender la expansión y propagación del COVID-19 (Bastos \& Cajueiro, 2020). Otro método para el análisis de las epidemias es el Susceptible-Exposed-Infectious-Recovered (SEIR) (Li, Graef, Wang, \& Karsai, 1999), este es un modelo dinámico que permite estudiar las enfermedades contagiosas teniendo en cuenta factores comportamentales de las poblaciones, este modelo permite simular los procesos de infección desde la fuente, los huéspedes, los reservorios y la transmisión a los humanos (De La Sen \& Alonso-Quesada, 2011; Liu \& Zhang, 2011). Además de adicionar variables de temporalidad como son el período de incubación de la enfermedad y los efectos de las medidas de contención. Este modelo ya ha sido utilizado en enfermedades infecciosas como el SARS, MERS y el COVID-19 (Peng, Yang, Zhang, Zhuge, \& Hong, 2020; Rao, Keshri, Mishra, $\&$ Panda, 2020).

El presente trabajo explora la construcción y aplicación de los modelos matemáticos SIR, SEIR y log-lineal con la finalidad de analizar y predecir 
el desarrollo y la evolución del SARS-CoV-2 en la República Dominicana. La adaptación de estos modelos al contexto dominicano, posibilitan explorar los índices de crecimiento del virus, la tasa de contagio y mortalidad, además de la predicción de diferentes escenarios sobre el final de la pandemia, a través de los datos oficiales disponibles hasta el 30 de abril del 2020.

\section{Materiales y métodos}

\section{Modelo Susceptible-Infectious-Recovered (SIR)}

Este modelo matemático posibilita estudiar las epidemias a través de la identificación de diferentes variables independientes y dependientes, estas ayudan a determinar los parámetros óptimos que permiten que el modelo se ajuste a los datos. El modelo estudia el flujo (figura 1) de las personas entre cuatro posibles estados, durante el brote de la enfermedad: Susceptible (S), Expuesto y Resistente (R) (Britton \& Pardoux, 2019), para esto utiliza las siguiente ecuación diferencial:

$$
\begin{gathered}
\frac{d S}{d t}=-\beta \cdot I \cdot \frac{S}{N} \quad(E c .1) \\
\frac{d I}{d t}=\beta . I \cdot \frac{S}{N}-\gamma \cdot I \quad(E c .2) \\
\frac{d R}{d t}=\gamma . I \quad(E c .3)
\end{gathered}
$$

Donde, $\mathrm{N}$ es la población total de la zona geográfica que está siendo estudiada; $S(t)$, es el número de persona susceptibles en un día; $\mathrm{I}(\mathrm{t})$ número de personas infectadas en el día; $\mathrm{R}(\mathrm{t})$ número de personas recuperadas en el día; $b$, es la cantidad de personas esperadas que una persona infectada puede infectar por día; $\mathrm{d}$, es el número de días que una persona infectada tiene y puede transmitir la enfermedad; $\mathrm{g}$, la proporción de infectados que se recuperan por día $(\mathrm{g}=1 / \mathrm{d})$ y $\mathrm{R}_{0}$ es el número total de personas que contagia una persona infectada $\left(\mathrm{R}_{0}=\mathrm{b} / \mathrm{g}\right)$.

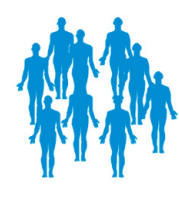

Susceptibles

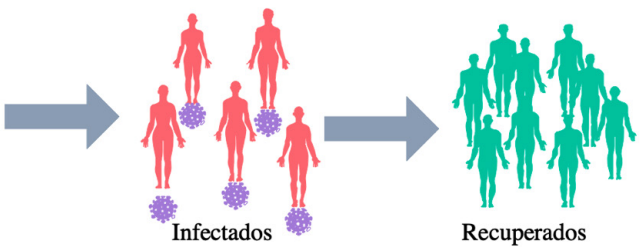

Figura 1. Representación gráfica del modelo SIR. El modelo estudia el proceso de propagación de una enfermedad infecciosa dentro de una población específica

Fuente: elaboración propia.

La primera ecuación (Ec.1) establece que el número de individuos susceptibles $(S)$ disminuye con el número de nuevos individuos recién infectados; la ecuación 2 (Ec.2) establece el número de individuos infecciosos (I) que aumenta con los individuos recién infectados, y la ecuación 3 (Ec.3) establece que el grupo de individuos recuperados (R) aumenta con el número de individuos que fueron infectados y que se recuperaron o murieron (Britton \& Pardoux, 2019; Soetewey, 2020).

Para implementar este modelo se usó el programa estadístico R (Free Software Foundation, 2016), basado en un método presentado por von Jouanne-Diedrich (2019) que realizó un análisis de los casos de COVID-19 en Alemania. Los códigos y datos fueron adaptados al contexto de la República Dominicana.

\section{Modelo SIR extendido (SEIR-E)}

Este modelo es una evolución del modelo SIR que incluye la exposición al patógeno como un nuevo estado dentro del modelo. Un individuo expuesto, es un individuo perteneciente al grupo de los susceptibles, que fue expuesto al virus, pero que todavía no puede transmitir la enfermedad. Este 
modelo también agrega dos variables: Muertos (D) y Recuperados (R), que son los estados a los que puede pasar una persona infectada después de varios días con la enfermedad (figura 2). Este modelo también permite el análisis de las personas que están en estado crítico, es decir, aquellos que van a necesitar de cuidados intensivos, así como la cantidad de camas en las unidades de cuidados intensivos (UCI) disponibles en el sistema de salud. El analizar estos números es de vital importancia para predecir las infraestructuras y cantidad de recursos sanitarios y de salud que serán necesarios para mitigar los efectos de la enfermedad (Amira et al., 2020; Li et al., 1999; J. Liu \& Zhang, 2011; Van Den Driessche, 2008).

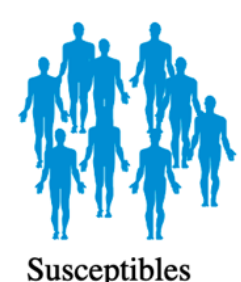

Susceptibles

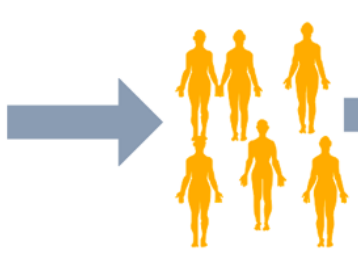

Expuestos
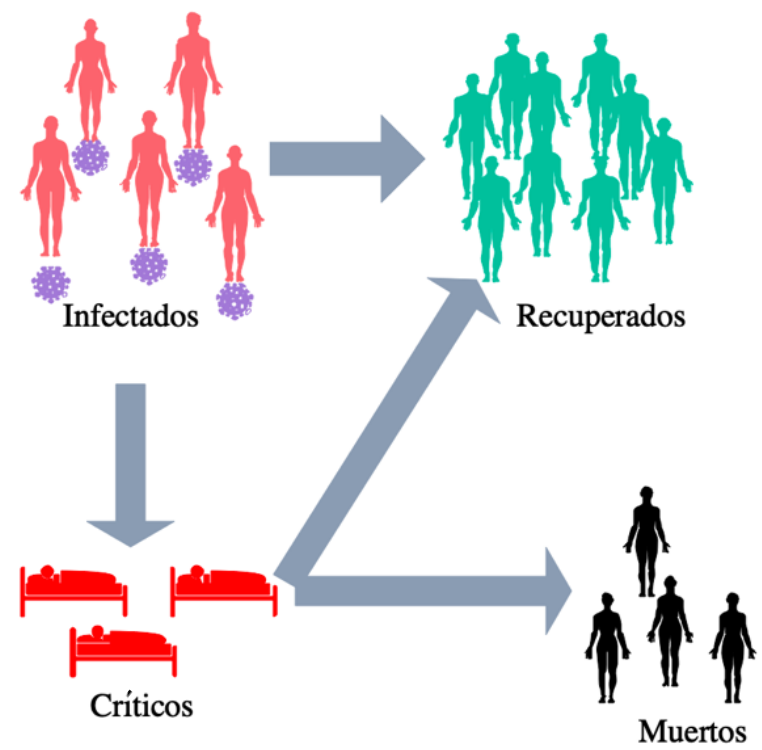

Figura 2. Representación gráfica del modelo SEIR-Extendido. El modelo presenta los diferentes estados de los individuos durante el brote y la propagación de una enfermedad

Fuente: elaboración propia.

Este modelo es representado por las siguientes ecuaciones:

$$
\begin{gathered}
\frac{d S}{d t}=-\beta(t) \cdot I \cdot \frac{S}{N} \quad(E c \cdot 4) \\
\frac{d S}{d t}=-\beta(t) \cdot I \cdot \frac{S}{N}-\delta \cdot E \cdot(E c .5) \\
\frac{d I}{d t}=\delta \cdot E-\frac{1}{12} \cdot p(I \rightarrow C) \cdot I-\gamma \cdot(1-p(I \rightarrow C)) \cdot I .
\end{gathered}
$$

donde, $\mathrm{N}$ es la población total de la zona geográfica que está siendo estudiada; $S(t)$, es el número de persona susceptibles en un día; $I(t)$ número de personas infectadas en el día; $\mathrm{R}(\mathrm{t})$ número de personas recuperadas en el día; $\mathrm{D}(\mathrm{t})$ número de personas fallecidas por días; $b$, es la cantidad de personas esperadas que una persona infectada puede contagiar por día; $\mathrm{D}$, es el número de días que una persona infectada tiene y puede transmitir la enfermedad; $\mathrm{g}$, la proporción de individuos que se que recuperan por día $(g=1 / D)$; $R_{0}$, es el número total de personas que puede contagiar una persona infectada $\left(\mathrm{R}_{0}=\mathrm{b} / \mathrm{g}\right) ; \mathrm{d}$, corresponde a la duración del período 
de incubación de la enfermedad; a, representa la tasa de mortalidad; $r$, es la tasa a la que mueren los individuos ( $=1 /$ días desde la infección hasta la muerte); C, representa la cantidad de individuos que necesitan cuidados intensivos; probabilidad de pasar de infectado a crítico y probabilidad de pasar de estado crítico a muerte (Froese, 2020; Van Den Driessche, 2008).

Basados en los informes y estadísticas actuales del COVID-19, fue estimado en 12 días (1/12), el parámetro en 7.5 días (1/7.5), y el número de días de un individuo para pasar de crítico a recuperado fue estimado en 6.5 (1/6.5) (Chen et al., 2020; Li et al., 2020; Wang et al., 2020; WHO, 2020a). Para implementar el modelo fue adaptado el método presentado por Froese (2020) utilizando el lenguaje de programación Python y Jupyter-notebook.

\section{Modelo log-lineal}

En la fase inicial del brote de una enfermedad, cuando se analizan los primeros casos y se representan en un gráfico; estos muestran una tendencia logarítmica lineal (el eje $y$ es una escala logarítmica y el eje $x$ sin transformación), lo permite que los datos pueden ser ajustados a una recta de la forma $m x+b$. Esta representación sugiere que pueden ser usados modelos log-lineal para modelar el crecimiento y el avance de la epidemia (Churches, 2020; Soetewey, 2020). Los modelos log-lineal, son modelos matemáticos que toman la forma de una función cuyo logaritmo es igual a una combinación lineal de los parámetros del modelo, esto permite que se puedan aplicar modelos de regresión lineal (Chung et al., 2016; Greenland, 1989).

El modelo lineal simple puede ser representado por la siguiente ecuación:

$$
\log (y)=r t+b
$$

Donde, y es la incidencia, $r$ es la tasa de crecimiento, t es el número de días desde un punto específico en el tiempo y b es la intercesión. Este modelo puede ser usado en el contexto del COVID-19 para generar dos modelos: 1) la fase de crecimiento (antes del pico); 2) la fase de descomposición (después del pico), que se ajustan a la curva de la epidemia (caso de incidencia) (Soetewey, 2020).

Para la implementación de este modelo fue utilizado el lenguaje estadístico R (Free Software Foundation, 2016). Para el modelado y ajustes de la curva fue utilizado el paquete incidence (Kamvar, Cai, Pulliam, Schumacher, \& Jombart, 2019) que forma parte del conjunto de paquetes de R Epidemic Consortium (RECON).

\section{Obtención de los datos}

Para la implementación de los modelos se recolectaron datos sobre el estado actual del COVID-19 en República Dominicana, a través de diferentes fuentes. El conjunto de datos con la cantidad de casos confirmados, números de muertos, nuevos casos diarios y cantidad de recuperados fueron obtenidos de los reportes sobre el virus, publicados diariamente por el Ministerio de Salud Pública de la República Dominicana (Ministerio de Salud Pública de República Dominicana, 2020) y de la base de datos de los casos globales de COVID-19 (Johns Hopkins University, 2020). Para generar los modelos de análisis, fue seleccionado un periodo de tiempo que comprende 60 días, desde el 1 de marzo 2020 (primer caso confirmado) hasta el 30 de abril de 2020. Los datos fueron obtenidos el día 30 de abril de 2020 .

El dato sobre la población total estimada de República Dominicana $(10,448,499)$ fue obtenido del informe de estimaciones y proyecciones de población total de la Oficina Nacional de Estadística de la República Dominicana (ONE) (2020) y los datos sobre la distribución de la pirámide poblacional estimada para República Dominicana, fueron tomados de Population Pyramid, (2019). 
Los datos sobre la cantidad de camas disponibles fueron obtenidos de la Organizacion de las Naciones Unidas (ONU) (2020), para obtener la cantidad de cama en las UCIs, se hizo una estimación, utilizando el promedio camas disponibles en otros países de América Latina que presentan un índice de desarrollo humano similar, utilizando los datos de la base de datos de la ONU.

\section{Resultados y discusión}

\section{Análisis de los datos oficiales}

Para entender el desarrollo de esta enfermedad en el país, se analizó su expansión desde el 1 de marzo al 30 de abril del 2020 (figura 3-A). A través del gráfico se puede verificar cómo la enfermedad ha tenido un crecimiento exponencial en el país; el 30 de abril corresponde al día que se alcanzó la barrera de los 7,000 casos confirmados y 300 muertes causadas por el virus. Los datos reflejados en esta figura no incluyen los casos asintomáticos y los casos que no han sido detectados y notificados oficialmente. Según las estimaciones realizadas (Russel, Hellewell, \& Abbot, 2020) al 30 de abril, en la República Dominicana solo el $16 \%$ de los casos estaban siendo detectados y notificados oficialmente, lo que indica que el $84 \%$ de los casos no habían sido detectados oficialmente (Ministerio de Salud Pública de República Dominicana, 2020). Según los reportes oficiales en mes de abril se habían detectado y notificado un total 7,288 casos de infecciones por COVID-19, lo que podría indicar alrededor de 38,000 casos que no habían sido detectados oficialmente. Estos números se pueden atribuir a la poca cantidad de pruebas para detectar el COVID-19 que están siendo realizadas en el país. Desde el reporte del primer caso, hasta el 30 de abril, se han hecho 28,446 pruebas, con un promedio de 900 pruebas por día, correspondiente a nueve pruebas por cada 100,000 habitantes, situación que dificulta el control de la propagación de la enfermedad.

La República Dominicana muestra un factor de crecimiento de las infecciones mayor a FC>1 (figura
3-B), alcanzando la última semana del mes de abril un promedio de 1.2, lo cual indica que la epidemia presenta un crecimiento exponencial y que la enfermedad no ha alcanzado el punto de inflexión, donde tenemos un $\mathrm{FC}<1$. Por lo que la cantidad de casos aumentará en las próximas semanas. Una de las posibles razones para tener este número, es que el país ha adoptado una estrategia de aislamiento social parcial, lo que permite que una cantidad significativa de personas estén expuestas al virus. La disminución pronunciada en la curva el día 7 de abril, puede deberse a un sesgo en los datos.

3-A

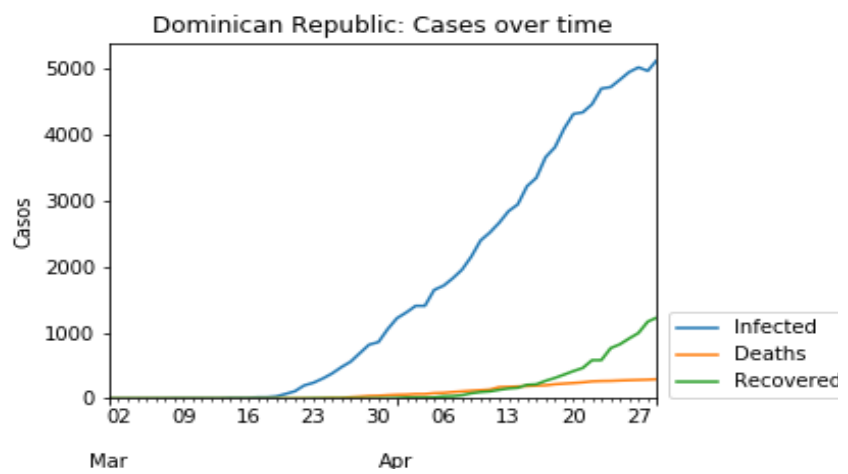

3-B

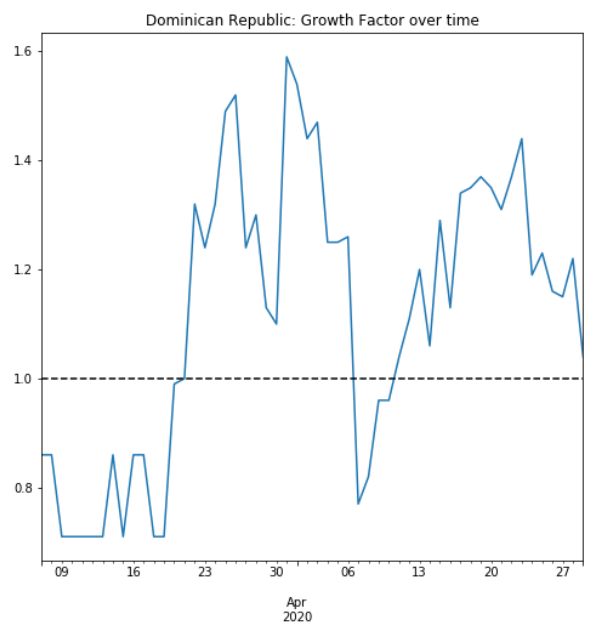

Figura 3. A- Acumulados de los casos confirmados, muertes e individuos recuperados durante el período del 1 de marzo al 30 de abril 2020. B- Tasa de infección $\left(\mathrm{R}_{0}\right)$ durante el periodo de tiempo del 1 de marzo al 30 de abril Fuente: elaboración propia. 


\section{Modelo SIR}

El modelo SIR simple fue aplicado a los datos, y los resultados indican que el país está dentro de la fase de crecimiento exponencial de la pandemia (figura 4-A). Se puede observar que durante los primeros
20 días de la pandemia ambas tendencias se superponen, pero a partir del día 22 se puede ver cómo la tendencia cambia, presentando una cantidad de casos confirmados mayor a la cantidad de casos proyectados por el modelo.

4-A

COVID-19 Proyección vs casos confirmados acumulado, Republica Dominicana (Rojo $=$ Ajuste del midelo SIR, Azul $=$ Casos confirmados)

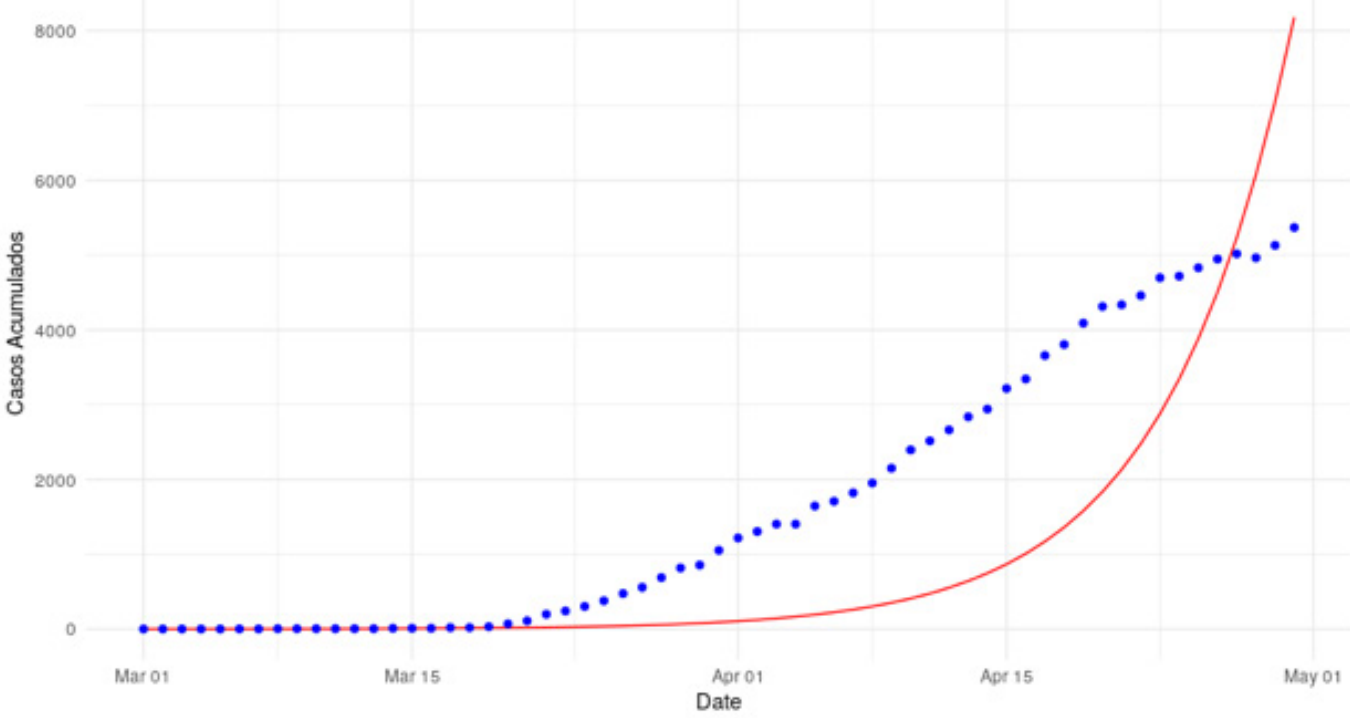

4-B

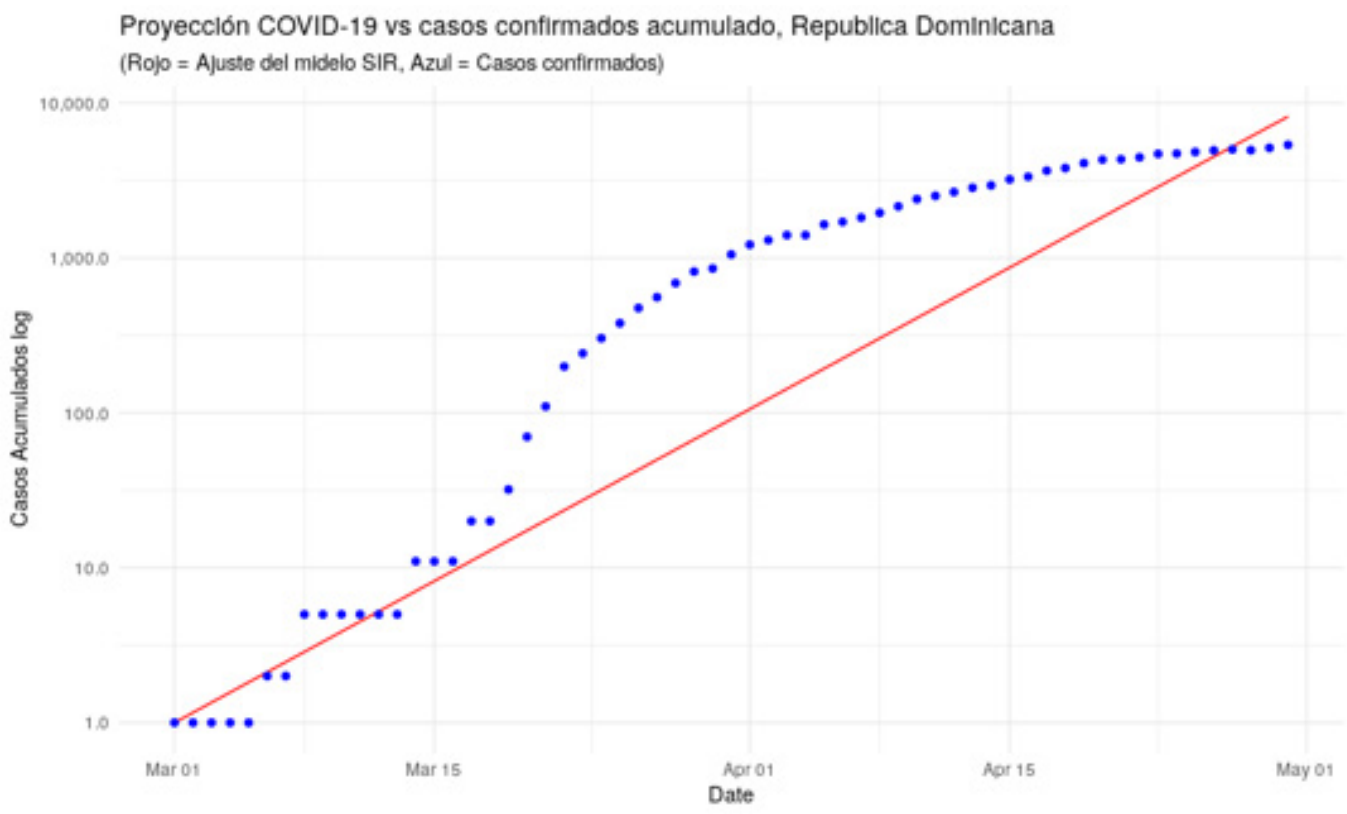

Figura 4. A- Resultado del modelo SIR, donde se comparan los casos reales versus los casos proyectados (Rojo: ajuste del modelo SIR, Azul: casos confirmados). B- Modelo SIR en escala logarítmica (Rojo: ajuste del modelo SIR, Azul: casos confirmados).

Fuente: elaboración propia. 
La figura (4-B), muestra el modelo en escala logarítmica, lo que permite observar las diferencias entre los datos proyectados y los casos confirmados y también se observa cómo los dos modelos difieren. El gráfico indica cómo los primeros cinco días de la pandemia los casos confirmados fueron menores a los proyectados, a partir de esta fecha tenemos una tendencia que sigue en aumento, en una tasa mayor a la exponencial. Con este modelo se obtuvo un $\mathrm{R}_{0}=1.3$, lo que indica que cada persona infectada puede estar contagiando a 1.3 personas.

Proyecclón de COVID-19 vs Casos confirmados acumulados, Republica DomInIcana

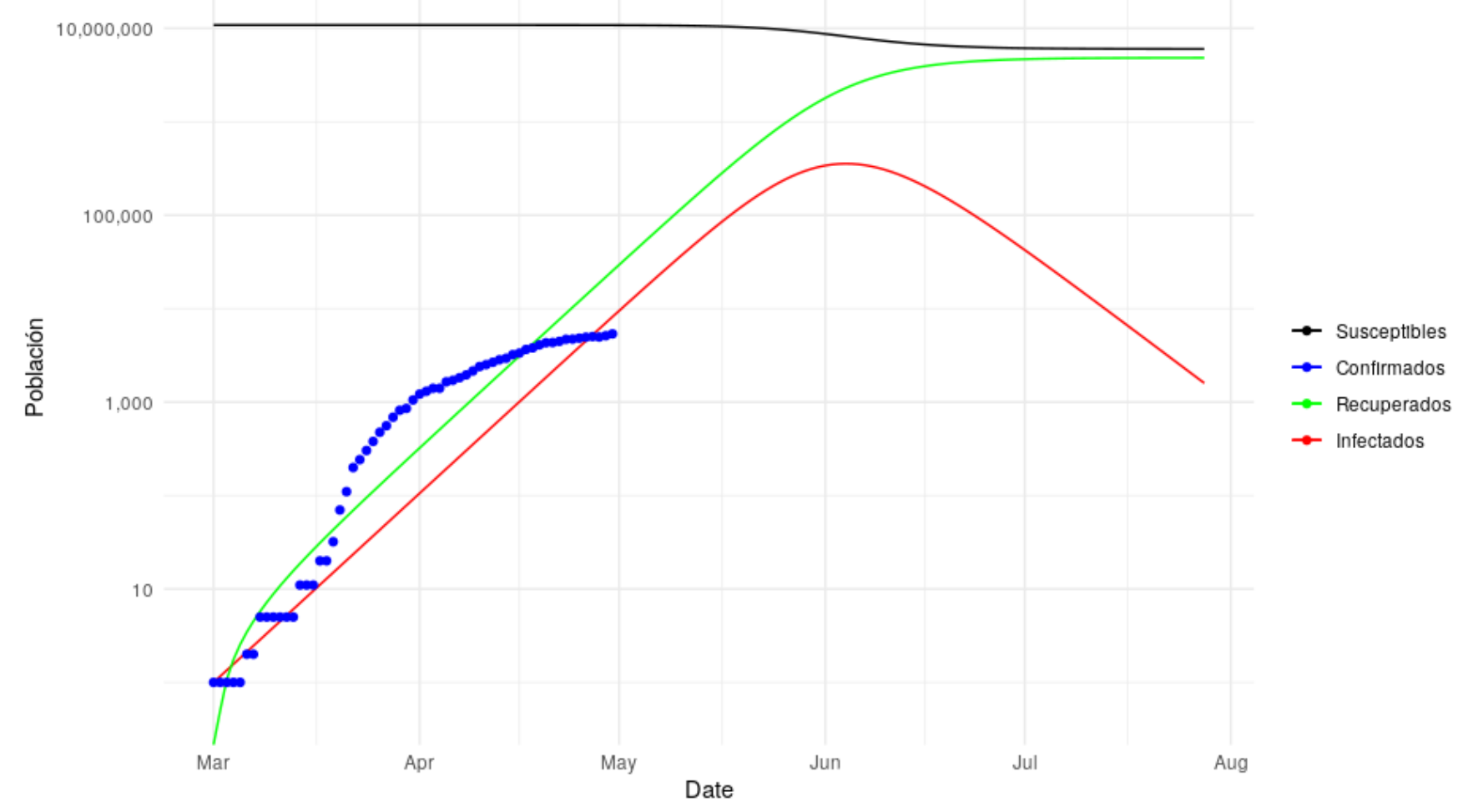

Figura 5. Proyección de COVID-19 vs. Casos confirmados acumulados, República Dominicana. Proyección avances y desarrollo del modelo SIR para 150 días, a partir del 30 de abril del 2020.

Fuente: elaboración propia.

La figura 5 muestra proyecciones del modelo SIR para los próximo 150 días, en el peor de los escenarios, es decir, que no se haya tomado ninguna medida de distanciamiento social, o que las medidas adoptadas no sean efectivas para mantener el respectivo distanciamiento. Como se puede observar, el punto de inflexión del modelo está ubicado próximo al día 6 de junio, con aproximadamente 355,000 infectados por el SARS-CoV-2. Tomando en cuenta la tasa de mortalidad en promedio $4.5 \%$ estimada por Roser,
Ritchie, Ortiz-Ospina, y Hansell (2020), podemos estimar una cantidad de óbitos en 16,015 y un total de 71,179 casos graves $(20 \%)$ al final de la primera fase de la pandemia. Basados en los datos obtenido por este modelo podemos verificar la importancia de tomar medidas que permitan contener la expansión del virus, debido a que bajo este escenario el sistema de salud no tendría capacidad para brindar atención adecuada a toda la población, además de que podría impactar la economía del país. 


\section{Modelo SEIR-Extendido}

Para entender mejor cómo es el proceso de propagación e incubación del virus en la población, se ha implementado el modelo SEIR extendido. Para ajustar el modelo a la realidad dominicana, el $\mathrm{R}_{0}$ fue establecido entre 1.04 y 1.10 , cálculo promedio obtenido a partir de los datos de MSP (2020) y Johns Hopkins University (2020).

\section{Modelo SEIR extendido}

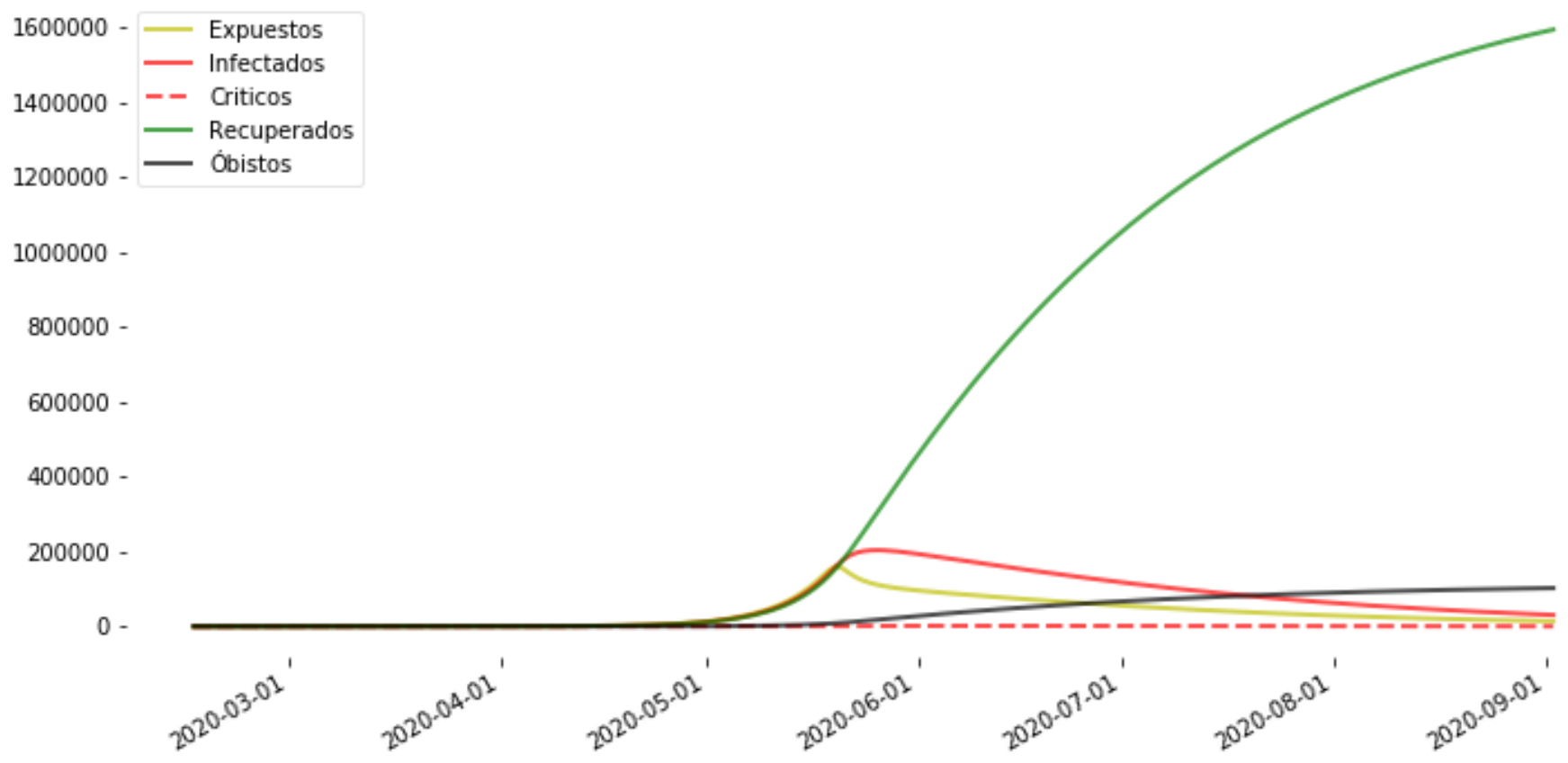

Figura 6. Proyección del modelo SEIR del avance y desarrollo de la pandemia en República Dominicana, para 200 días a partir del 30 de abril 2020

Fuente: elaboración propia.

La figura 6 muestra las proyecciones del modelo para los próximos 200 días, según los datos, el pico de la curva de crecimiento del virus será alcanzado aproximadamente entre los días 31 de mayo al 4 de junio 2020, con un aproximado de 200,000 infectados por el virus, de estos el $9 \%$ necesitaría cuidados especiales en la UCI y el $79 \%$ de los pacientes en la UCI no superarían la enfermedad. El número total de fallecidos relacionados con el virus estaría próximo a los 1,600 casos. El modelo proyecta una tasa de fatalidad de máxima del $6 \%$ y un $\mathrm{R}_{0}$ máximo de 3 .

\section{Modelo log-lineal}

Para entender el crecimiento epidemiológico del virus en la República Dominicana, los datos fueron modelados con el modelo log-lineal. La figura 7-A, muestra el desarrollo de la enfermedad (casos confirmados) durante los 60 días que fueron analizados en este estudio, alcanzando el pico más pronunciado el día 16 de abril, a partir de este día podemos observar una leve disminución de los casos confirmados, aunque no se puede inferir que se haya 
iniciado el descenso de la curva, debido a que la tasa que la epidemia todavía se mantiene en fase de crecide infección permanece entre 1.2 y 1.3, lo que indica miento exponencial.

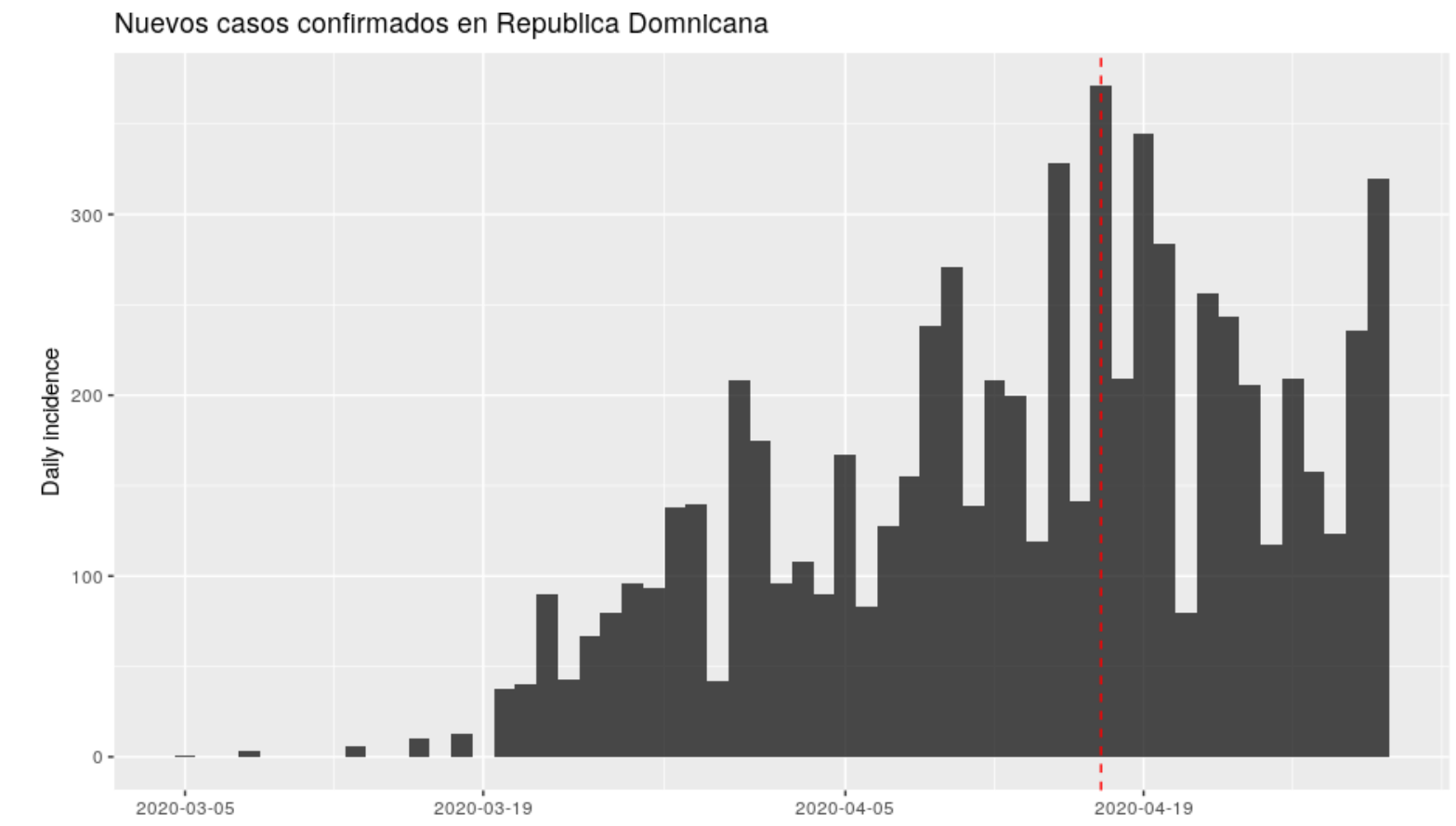

Incldencla observada y modelada de los casos deCOVID-19

Republica Dominicana, 2020

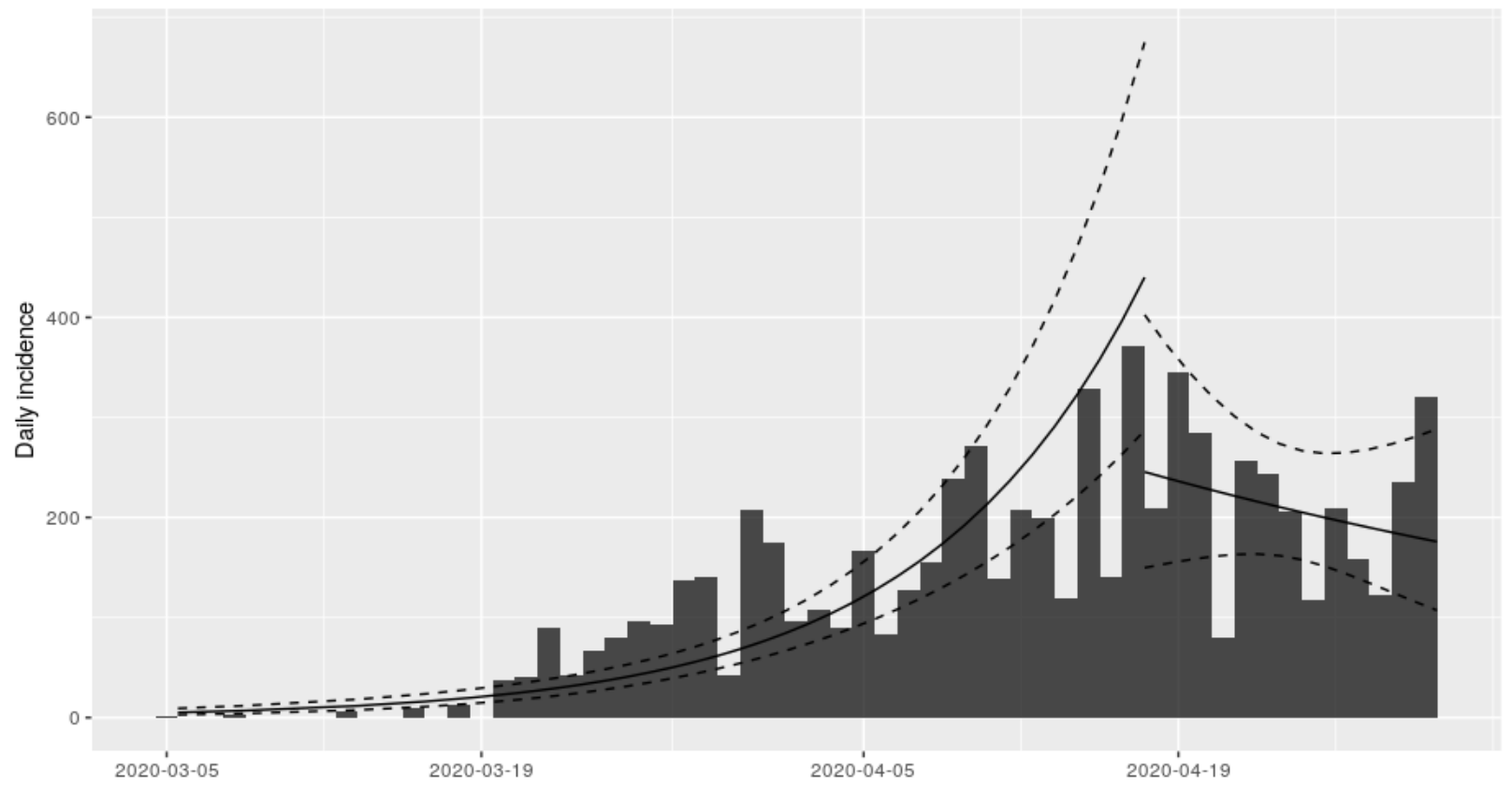

Figura 7. A. Crecimiento epidemiológico durante el período del 1 de marzo al 30 de abril. B. Tendencias de pandemia en República Dominicana durante el período del 1 de marzo al 30 de abril.

Fuente: elaboración propia. 
La figura 7-B muestra un ajuste del modelo antes y después del pico; ante del pico la tasa de crecimiento estaba en 0.10 y la tasa de reducción después del pico fue solo de -0.026. Esta tasa de crecimiento representa un tiempo de duplicación de los casos de 6.7 días.

Basada en la incidencia de casos diarios reportado durante el intervalo de tiempo analizado, se hizo la proyección de los casos esperados para los próximos meses. La figura 8 muestra una proyección de casos para los próximos cuatro meses. Como se puede observar en la imagen, los caso confirmados comienzan a disminuir a partir de la segunda mitad del mes de mayo, llegando a menos de 50 casos por día, a partir del inicio del mes de julio.

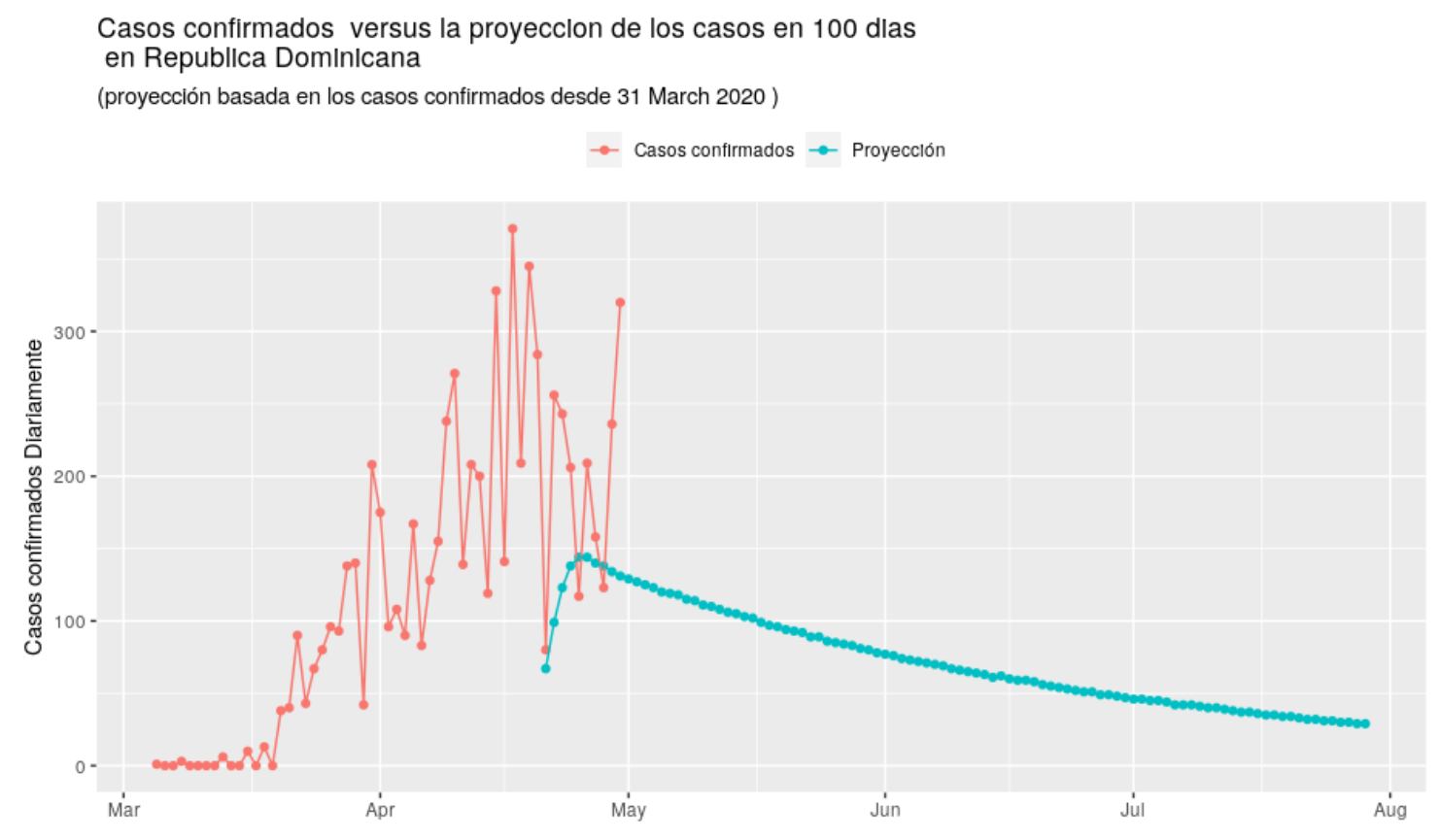

Figura 8. Proyecciones del modelo de incidencia de los casos confirmado para cuatro (4) meses, a partir del 30 de abril 2020

Fuente: elaboración propia.

\section{Conclusiones}

La pandemia del COVID-19 se ha extendido por la mayoría de los países del mundo, obligando a que los gobiernos tomen medidas sin precedentes, para mitigar la propagación del virus. De este modo se ha generado una gran presión en los sistemas de salud de estos países, los cuales no estaban preparados para una crisis de esta magnitud; ha quedado evidenciado que los recursos y la infraestructura sanitarias son insuficiente para manejar el gran volumen de casos.
En este trabajo fueron implementados los modelos SIR, SEIR y log-lineal, para modelar los posibles escenarios y evaluar el impacto de la pandemia en República Dominicana, además del efecto de las medidas tomadas por las autoridades. Los modelos son esenciales para entender el desarrollo y la evolución de las enfermedades y epidemias proyectando los posibles escenarios que pueden suceder, lo que permite que diversas decisiones puedan ser tomadas por las autoridades competentes con el fin de contener la propagación del patógeno. Las esti- 
maciones obtenidas por los modelos no difieren mucho de otras estimaciones ya realizadas para el país (Luo, 2020), que estiman la disminución de los casos entre mayo y junio. Los resultados obtenidos en este estudio pueden ser utilizados para la toma de decisiones que permitan una reactivación de la sociedad de forma gradual, y de este modo disminuir los riesgos de una nueva ola de contagios.

Las aproximaciones presentadas a partir de los datos y modelos obtenidos no necesariamente predicen por completo el comportamiento de la pandemia, debido que esto pueden ser afectados por diversos factores y variables (sociales, económicas y culturales) que no pueden ser introducidos dentro del modelo, además la variación de los datos es constante, lo que puede cambiar de forma drástica las tendencias y proyecciones. Otro factor importante para la construcción de los modelos es la calidad y la disponibilidad de los datos. En la República Dominicana hay dificultad para acceder a los datos, además, existen variables que no son recolectadas y medidas por los órganos gubernamentales, lo que implicó una de las mayores limitaciones de este estudio. Por esta razón, no fue posible proyectar modelos robustos que pudieran medir el impacto del aislamiento social en las diferentes regiones y ciudades del país.

Se debe resaltar que los resultados deben interpretarse con cuidado, ya que las proyecciones en estas etapas de desarrollo y crecimiento de la curva epidemiológica dependen mucho de la calidad de los datos, pequeños cambios en los valores observados pueden producir grandes variaciones de tendencia en las proyecciones.

\section{Disponibilidad de los datos y códigos}

Los datos y códigos usados en este estudio están disponibles en https://github.com/edianfranklin

\section{Agradecimientos}

El presente trabajo fue desarrollado con el apoyo del Ministerio de Educación Superior Ciencia y
Tecnología de la República Dominicana (MESCyT), a través del Fondo Nacional de Innovación y Desarrollo Científico-Tecnológico (FONDOCYT), subvención No. 2018-2018-2B4-157.

Asimismo, contó con el apoyo de la Coordenação de Aperfeiçoamento de Pessoal de Nível Superior Brasil (CAPES) - Código de Financiamiento 001.

\section{Referencias}

Amira, F., Hamzah, B., Lau, C. H., Nazri, H., Ligot, D. V., Lee, G., ... Salunga, R. E. (2020). Outbreak Data Analysis and Prediction. Bull World Health Organ. E-Pub. Recuperado de https://doi.org/10.2471/BLT.20.251561

Andersen, K. G., Rambaut, A., Lipkin, W. I., Holmes, E. C., \& Garry, R. F. (2020). The proximal origin of SARS-CoV-2. Nature Medicine. Nature Research. Recuperado de https://doi. org/10.1038/s41591-020-0820-9

Bastos, S. B., \& Cajueiro, D. O. (2020). Modeling and forecasting the early evolution of the Covid-19 pandemic in Brazil.

Bootsma, M. C. J., \& Ferguson, N. M. (2007). The effect of public health measures on the 1918 influenza pandemic in U.S. cities. Proceedings of the National Academy of Sciences of the United States of America, 104(18), 7588-7593. Recuperado de https://doi. org/10.1073/pnas.0611071104

Britton, T., \& Pardoux, E. (2019). SIR Epidemics on Configuration Model Graphs. In Lecture Notes in Mathematics (Vol. 2255, pp. 263298). Springer. Recuperado de https://doi. org/10.1007/978-3-030-30900-8_10

Chan, J. F. W., Yuan, S., Kok, K. H., To, K. K. W., Chu, H., Yang, J., ... Yuen, K. Y. (2020). A familial cluster of pneumonia associated with the 2019 novel coronavirus indicating person-to-person transmission: a study of a family cluster. The Lancet, 395(10223), 514523. Recuperado de https://doi.org/10.1016/ S0140-6736(20)30154-9 
Chen, N., Zhou, M., Dong, X., Qu, J., Gong, F., Han, Y., ... Zhang, L. (2020). Epidemiological and clinical characteristics of 99 cases of 2019 novel coronavirus pneumonia in Wuhan, China: a descriptive study. The Lancet, 395(10223), 507513. Recuperado de https://doi.org/10.1016/ S0140-6736(20)30211-7

Chung, C. L., Huang, K. J., Chen, S. Y., Lai, M. H., Chen, Y. C., \& Kuo, Y. F. (2016). Detecting Bakanae disease in rice seedlings by machine vision. Computers and Electronics in Agriculture. Recuperado de https://doi.org/10.1016/j. compag.2016.01.008

Churches, T. (2020). Analysing COVID-19 (2019$\mathrm{nCoV}$ ) outbreak data with $\mathrm{R}$ - part 1 . Recuperado de https://timchurches.github.io/blog/ posts/2020-02-18-analysing-covid-19-2019ncov-outbreak-data-with-r-part-1/

De La Sen, M., \& Alonso-Quesada, S. (2011). Vaccination strategies based on feedback control techniques for a general SEIR-epidemic model. Applied Mathematics and Computation, 218(7), 3888-3904. Recuperado de https:// doi.org/10.1016/j.amc.2011.09.036

Dong, E., Du, H., \& Gardner, L. (2020). An interactive web-based dashboard to track COVID-19 in real time. The Lancet Infectious Diseases. Lancet Publishing Group. Recuperado de https://doi.org/10.1016/ S1473-3099(20)30120-1

Forster, P., Forster, L., Renfrew, C., \& Forster, M. (2020). Phylogenetic network analysis of SARS-CoV-2 genomes. Proceedings of the National Academy of Sciences, 117(17), 202004999. Recuperado de https://doi.org/10.1073/pnas. 2004999117

Free Software Foundation, I. (2016). GNU R. Free Software Foundation, Inc.

Froese, H. (2020). Modelling Coronaviurs: Fit your model to real-world data |Towards Data Science. Recuperado de https://towardsdatascience. com/infectious-disease-modelling-fit-your-model-to-coronavirus-data-2568e672dbc7
Greenland, S. (1989). Modeling and variable selection in epidemiologic analysis. American Journal of Public Health, 79(3), 340-349. Recuperado de https://doi.org/10.2105/AJPH. 79.3.340

Huang, C., Wang, Y., Li, X., Ren, L., Zhao, J., Hu, Y., ... Cao, B. (2020). Clinical features of patients infected with 2019 novel coronavirus in Wuhan, China. The Lancet, 395(10223), 497-506. Recuperado de https://doi.org/ 10.1016/S0140-6736(20)30183-5

Hui, D. S., I Azhar, E., Madani, T. A., Ntoumi, F., Kock, R., Dar, O., ... Petersen, E. (2020). The continuing 2019-nCoV epidemic threat of novel coronaviruses to global health - The latest 2019 novel coronavirus outbreak in Wuhan, China. International Journal of Infectious Diseases. Elsevier B.V. Recuperado de https://doi.org/10.1016/j.ijid.2020.01.009

Johns Hopkins University. (2020). Global Cases of COVID-19 by the Center for Systems Science and Engineering (CSSE) at Johns Hopkins University. Coronavirus Resource Center, 17, 2020.

Kamvar, Z. N., Cai, J., Pulliam, J. R. C., Schumacher, J., \& Jombart, T. (2019). Epidemic curves made easy using the $\mathrm{R}$ package incidence. F1000Research, 8. Recuperado de https:// doi.org/10.12688/f1000research.18002.1

Kermack, W. O., \& McKendrick, A. G. (1927). A contribution to the mathematical theory of epidemics. Proceedings of the Royal Society of London. Series A, Containing Papers of a Mathematical and Physical Character, 115(772), 700-721.

Li, M. Y., Graef, J. R., Wang, L., \& Karsai, J. (1999). Global dynamics of a SEIR model with varying total population size. Mathematical Biosciences, 160(2), 191-213. Recuperado de https://doi. org/10.1016/S0025-5564(99)00030-9

Li, Q., Guan, X., Wu, P., Wang, X., Zhou, L., Tong, Y., ... Feng, Z. (2020). Early transmission dynamics in Wuhan, China, of novel coronavi- 
rus-infected pneumonia. New England Journal of Medicine. Massachussetts Medical Society. Recuperado de https://doi.org/10.1056/NEJMoa2001316

Lin, F., Muthuraman, K., \& Lawley, M. (2010). An optimal control theory approach to non-pharmaceutical interventions. BMC Infectious Diseases, 10(1), 32. Recuperado de https:// doi.org/10.1186/1471-2334-10-32

Liu, J., \& Zhang, T. (2011). Epidemic spreading of an SEIRS model in scale-free networks. Communications in Nonlinear Science and Numerical Simulation, 16(8), 3375-3384. Recuperado de https://doi.org/10.1016/j. cnsns.2010.11.019

Liu, Y., Gayle, A. A., Wilder-Smith, A., \& Rocklöv, J. (2020). The reproductive number of COVID-19 is higher compared to SARS coronavirus. Journal of Travel Medicine, 27(2). Recuperado de https://doi.org/10.1093/jtm/ taaa021

Luo, J. (2020). Predictive Monitoring of COVID-19 Life Cycle Curves. Recuperado de https:// ddi.sutd.edu.sg/

Markel, H., Stern, A. M., Navarro, J. A., Michalsen, J. R., Monto, A. S., \& DiGiovanni, C. (2006). Nonpharmaceutical influenza mitigation strategies, US communities, 19181920 pandemic. Emerging Infectious Diseases, 12(12), 1961-1964. https://doi.org/10.3201/ eid1212.060506

Ministerio de Salud Pública de República Dominicana -MSP (2020). COVID-19 - Ministerio de Salud Pública. Recuperado de https:// www.msp.gob.do/web/?page_id=6948

Oficina Nacional de Estadística de la República Dominicana -ONE. (2020). Total, Estimaciones y proyecciones de la poblacion. Recuperado de https://www.one.gob.do/

Organizacion de las Naciones Unidas -ONU. (2020). UNdata. Recuperado de https:// data.un.org/
Organización Panamericana de la Salud - OPS. (2020). OPS/OMS Rep. Dom. - Primer caso importado del nuevo coronavirus confirmado en República Dominicana. Recuperado de https:// www.paho.org/dor/index.php?option=com_ content\&view=article\&id=3240:primer-caso-importado-del-nuevo-coronavirus-confirmado-en-republica-dominicana\&Itemid $=362$

Peng, L., Yang, W., Zhang, D., Zhuge, C., \& Hong, L. (n.d.). Epidemic analysis of COVID-19 in China by dynamical modeling. Recuperado de http://www.nhc.gov.cn/

Population Pyramid. (2019). Dominican Republic 2019. Recuperado de https://www.populationpyramid.net/dominican-republic/2019/

Rao, Y. S., Keshri, A. K., Mishra, B. K., \& Panda, T. C. (2020). Distributed denial of service attack on targeted resources in a computer network for critical infrastructure: A differential e-epidemic model. Physica A: Statistical Mechanics and Its Applications, 540. Recuperado de https:// doi.org/10.1016/j.physa.2019.123240

Riley, S., \& Ferguson, N. M. (2006). Smallpox transmission and control: Spatial dynamics in Great Britain. Proceedings of the National Academy of Sciences of the United States of America, 103(33), 12637-12642. Recuperado de https://doi.org/10.1073/pnas.0510873103

Riley, S., Fraser, C., Donnelly, C. A., Ghani, A. C., Abu-Raddad, L. J., Hedley, A. J., ... Anderson, R. M. (2003). Transmission dynamics of the etiological agent of SARS in Hong Kong: Impact of public health interventions. Science, 300(5627), 1961-1966. Recuperado de https:// doi.org/10.1126/science. 1086478

Rios Montanez,A. M. (2020). Cases of coronavirus in Latin America 2020 | Statista. Recuperado de https://www.statista.com/statistics/1101643/ latin-america-caribbean-coronavirus-cases/

Roser, M., Ritchie, H., Ortiz-Ospina, E., \& Hasell, J. (2020). Coronavirus Pandemic (COVID-19) - Statistics and Research - Our World in Data. Recuperado de https://ourworldindata.org/ mortality-risk-covid?country=DOM 
Russel, T., Hellewell, J., \& Abbot, S. (2020). Using a delay-adjusted case fatality ratio to estimate under-reporting. Available at the Centre for Mathematical Modelling of Infectious Diseases Repository, Here.

Sanche, S., Lin, Y. T., Xu, C., Romero-Severson, E., Hengartner, N., \& Ke, R. (2020). The Novel Coronavirus, 2019-nCoV, is Highly Contagious and More Infectious Than Initially Estimated. MedRxiv, 2020.02.07.20021154. Recuperado de https://doi.org/10.1101/ 2020.02.07.20021154

Soetewey, A. (2020). COVID-19 in Belgium - Stats and R. Recuperado de https://www.statsandr. com/blog/covid-19-in-belgium/

Tang, B., Bragazzi, N. L., Li, Q., Tang, S., Xiao, Y., \& Wu, J. (2020). An updated estimation of the risk of transmission of the novel coronavirus (2019-nCov). Infectious Disease Modelling, 5, 248-255. Recuperado de https:// doi.org/10.1016/j.idm.2020.02.001

Tang, B., Wang, X., Li, Q., Bragazzi, N. L., Tang, S., Xiao, Y., \& Wu, J. (2020). Estimation of the Transmission Risk of the 2019-nCoV and Its Implication for Public Health Interventions. Journal of Clinical Medicine, 9(2), 462. Recuperado https://doi.org/10.3390/ jcm 9020462

Van Den Driessche, P. (2008). Deterministic compartmental models: Extensions of basic models. Lecture Notes in Mathematics, 1945, 147-157. Recuperado de https://doi.org/10.1007/9783-540-78911-6_5

Viceconte, G., \& Petrosillo, N. (2020). Covid-19 R0: Magic number or conundrum? Infectious Disease Reports. Page Press Publications. Recuperado https://doi.org/10.4081/idr.2020.8516

von Jouanne-Diedrich, H. K. (2019). Epidemiology: How contagious is Novel Coronavirus (2019$\mathrm{nCoV})$ ? Recuperado de https://blog.ephorie. de/epidemiology-how-contagious-is-novel-coronavirus-2019-ncov

Wang, C., Horby, P. W., Hayden, F. G., \& Gao, G. F. (2020). A novel coronavirus outbreak of global health concern. The Lancet. Lancet Publishing Group. Recuperado de https://doi. org/10.1016/S0140-6736(20)30185-9

Wang, D., Hu, B., Hu, C., Zhu, F., Liu, X., Zhang, J., ... Peng, Z. (2020). Clinical Characteristics of 138 Hospitalized Patients with 2019 Novel Coronavirus-Infected Pneumonia in Wuhan, China. JAMA - Journal of the American Medical Association, 323(11), 1061-1069. Recuperado de https://doi.org/10.1001/ jama.2020.1585

WHO, W. H. O. (2020a). Report of the who-china joint mission on coronavirus disease 2019 (covid-19). Geneva.

WHO, W. H. O. (2020b). Statement on the second meeting of the International Health Regulations (2005) Emergency Committee regarding the outbreak of novel coronavirus (2019-nCoV).

Wu, F., Zhao, S., Yu, B., Chen, Y. M., Wang, W., Song, Z. G., ... Zhang, Y. Z. (2020). A new coronavirus associated with human respiratory disease in China. Nature, 579(7798), 265269 Recuperado de https://doi.org/10.1038/ s41586-020-2008-3

Zhao, S., Lin, Q., Ran, J., Musa, S. S., Yang, G., Wang, W., ... Wang, M. H. (2020). Preliminary estimation of the basic reproduction number of novel coronavirus (2019-nCoV) in China, from 2019 to 2020: A data-driven analysis in the early phase of the outbreak. International Journal of Infectious Diseases, 92, 214-217. https://doi.org/10.1016/j. ijid.2020.01.050

Zhao, S., Musa, S. S., Lin, Q., Ran, J., Yang, G., Wang, W., ... Wang, M. H. (2020). Estimating the Unreported Number of Novel Coronavirus (2019-nCoV) Cases in China in the First Half of January 2020: A Data-Driven Modelling Analysis of the Early Outbreak. Journal of Clinical Medicine, 9(2), 388. Recuperado de https://doi.org/10.3390/jcm9020388 
Zhou, P., Yang, X. Lou, Wang, X. G., Hu, B., Zhang, L., Zhang, W., ... Shi, Z. L. (2020). A pneumonia outbreak associated with a new coronavirus of probable bat origin. Nature, 579(7798), 270-273. Recuperado de https:// doi.org/10.1038/s41586-020-2012-7
Zhu, N., Zhang, D., Wang, W., Li, X., Yang, B., Song, J., ... Tan, W. (2020). A novel coronavirus from patients with pneumonia in China, 2019. New England Journal of Medicine, 382(8), 727-733. Recuperado de https:// doi.org/10.1056/NEJMoa2001017 